\title{
Three-phonon relaxation in isotropic and anisotropic phonon systems of liquid helium at different pressures
}

\author{
I.N. Adamenko ${ }^{1,2}$, Yu.A. Kitsenko ${ }^{3}$, K.E. Nemchenko ${ }^{1}$, V.A. Slipko ${ }^{1}$, \\ and A.F.G. Wyatt ${ }^{4}$ \\ ${ }^{1}$ Karazin Kharkov National University, 4 Svobody Sq., Kharkov 61077, Ukraine \\ E-mail: i.n.adamenko@mail.ru \\ ${ }^{2}$ Electro-Physical Scientific and Technical Centre National Academy of Sciences of Ukraine \\ 28 Chernyshevsky St., Kharkov 61002, Ukraine \\ ${ }^{3}$ Akhiezer Institute for Theoretical Physics National Scientific Center «Kharkov Institute of Physics \\ and Technology» National Academy of Sciences of Ukraine \\ 1 Academicheskaya St., Kharkov 61108, Ukraine \\ ${ }^{4}$ School of Physics, University of Exeter, Exeter, EX4 4QL, UK
}

Received September 24, revised October 8, 2004

\begin{abstract}
Starting from the kinetic equation for phonons in superfluid helium, expressions for the rates of three-phonon scattering in isotropic and anisotropic phonon systems were obtained for different pressures. These expressions are valid in the whole range of energies where three-phonon processes are allowed. Limiting cases were analysed and compared with the results of previous theoretical investigations. The obtained pressure and angular dependence of three phonon scattering rate allows one to explaine of the experimental data on interaction of phonon pulses.
\end{abstract}

PACS: 67.40.Db, 67.40.Fd, 67.90.+z

\section{Introduction}

The dispersion relation of superfluid helium (He II) has an important role in determining the interactions in its phonon systems. The dispersion relation can be written as

$$
\varepsilon(p)=c p(1+\psi(p)),
$$

where $\varepsilon$ is the phonon energy, $p$ is its momentum, $c$ is the sound velocity in He II, $\psi(p)<<1$ is the deviation of the spectrum from linearity, which is small but nevertheless completely determines the mechanisms of phonon interactions. We note that, as well as at $p=0$, there is a critical momentum $p_{c}$ at which the function $\psi(p)$ is zero:

$$
\psi(0)=\psi\left(p_{c}\right)=0 .
$$

When $p<p_{c}$ the function $\psi>0$ (anomalous dispersion). In this case the conservation laws of energy and momentum allow processes which do not conserve the numbers of phonons. The fastest of these processes is the three-phonon process in which one phonon decays into two or two interacting phonons combine into one phonon. This paper is devoted to the consideration of such processes.

When $p>p_{c}$ the function $\psi<0$. In this case the dispersion is normal and three-phonon processes are prohibited by the conservation laws of energy and momentum. Then the fastest processes are four-phonon processes.

Phonon systems can be isotropic or anisotropic, depending on whether or not there is a special direction in momentum space. Isotropic phonon systems can be easily created experimentally by heating a volume of fluid He II. These systems have been intensively investigated theoretically over several decades. Anisotropic phonon systems are a relatively new area of low-temperatures physics. In experiments they are created by a plane heater which injects a pulse of phonons into superfluid He II (see, for example, [1-3]).

The stimulus for carrying out this investigation were the experiments $[4,5]$, where the interaction of phonon pulses was observed. These experiments have 
shown that pulses interact at pressures from 0 to 12 bar, and when the pressure is higher, for example, at $18 \mathrm{bar}$, there is no interaction. Also in these experiments the dependence of the interaction on the angle between the axes of the pulses was investigated. It was found that pulses interact strongly if the angle between their axes is $8.8^{\circ}$, but at $46^{\circ}$ there is no interaction.

The purpose of this paper is to calculate the rate of three-phonon processes in isotropic and anisotropic phonon systems of superfluid helium at different pressures and to compare the results of the calculations with experimental data $[4,5]$.

\section{The main characteristics of three-phonon processes}

The dispersion relation (1) is very important for studying three-phonon processes. As the behavior at different pressures will be considered in this paper, it is necessary to take into account the pressure dependence of the parameters in expression (1).

For calculations in this paper we use a simple analytical approximation of the function $\psi$, which nevertheless describes all the available experimental data (see, for example, [6,7]) rather well:

$$
\psi(p, P)=4 \psi_{\max }(P) \frac{p^{2}}{p_{c}^{2}(P)}\left(1-\frac{p^{2}}{p_{c}^{2}(P)}\right),
$$

where $p_{c}(P)$ is the critical value of momentum, which depends on pressure $P$ and $\psi_{\max }(P)$ is the maximum value of the function $\psi(p, P)$ which is reached when $p=p_{c} / \sqrt{2}$. The expression for function $\psi_{\max }(P)$ can be written as

$$
\begin{gathered}
\psi_{\text {max }}(P)=4.551 \cdot 10^{-2}-5.537 \cdot 10^{-3} P+ \\
+2.155 \cdot 10^{-4} P^{2}-2.415 \cdot 10^{-6} P^{3} .
\end{gathered}
$$

Expression (4) is obtained from an analysis of Refs. 6,8 and 9 .

The expression (3) is valid in the region $p<p_{c}$ for any pressures up to $P=19$ bar, the pressure at which the dispersion becomes normal. To calculate the rate of three phonon processes in isotropic and anisotropic phonon systems at different pressures, it is necessary to have the dependences $c(P)$ and $\tilde{p}_{c}(P)=$ $=c(P) p_{c}(P) / k_{B}$, which can be written as

$$
\begin{gathered}
c(P)=10^{2}\left(238.3+7.33146 P-0.09141 P^{2}\right), \\
\tilde{p}_{c}(P)=10-0.20915 P-0.01669 P^{2} .
\end{gathered}
$$

Expression (5) is obtained from an analysis of experimental data [10] and (6) from [7-9,11,12]. Here and below values of the variables are given in CGS units except that $\tilde{p}_{c}$ is in Kelvin and pressure in bar.

We write out the dependences of the density $\rho(P)$ and Grüneisen constant $u=(\rho / c)(\partial c / \partial \rho)$ on pressure, as they will be necessary for our further calculations:

$$
\begin{gathered}
\rho(P)=0.14513+0.00171 P- \\
-3.7661 \cdot 10^{-5} P^{2}+5.29924 \cdot 10^{-7} P^{3}, \\
u(P)=2.84-0.05537 P+0.00201 P^{2}- \\
-3.24998 \cdot 10^{-5} P^{3} .
\end{gathered}
$$

These dependences were found from experimental data of Ref. 10 as well as expression (5).

It has long been realized that three-phonon processes cannot take place all the way to the critical momentum $p_{c}$. From the conservation laws of momentum and energy which must apply in such processes:

$$
\begin{gathered}
\mathbf{p}^{\prime}+\mathbf{p}^{\prime \prime}=\mathbf{p}, \\
\varepsilon\left(p^{\prime}\right)+\varepsilon\left(p^{\prime}\right)=\varepsilon(p),
\end{gathered}
$$

and also from expression (1) we obtain restrictions on the momenta of the interacting phonons. Starting from (1), (9) and (10), we calculate the angles between phonons with momenta $\mathbf{p}^{\prime}, \mathbf{p}^{\prime \prime}$ and $\mathbf{p}, \mathbf{p}^{\prime}$. As a result we have:

$\zeta_{p^{\prime} p^{\prime \prime}}=\frac{p^{\prime}+p^{\prime \prime}}{p^{\prime} p^{\prime \prime}}\left[\left(p^{\prime}+p^{\prime \prime}\right) \psi\left(p^{\prime}+p^{\prime \prime}\right)-p^{\prime} \psi\left(p^{\prime}\right)-p^{\prime \prime} \psi\left(p^{\prime \prime}\right)\right]$,

$\zeta_{p p^{\prime}}=\frac{p-p^{\prime}}{p p^{\prime}}\left[p \psi(p)-p^{\prime} \psi\left(p^{\prime}\right)-\left(p-p^{\prime}\right) \psi\left(p-p^{\prime}\right)\right]$,

where $\zeta_{p^{\prime} p^{\prime \prime}}=1-\cos \theta_{p^{\prime} p^{\prime \prime}}, \quad \zeta_{p p^{\prime}}=1-\cos \theta_{p p^{\prime}}$ and $\theta_{p^{\prime} p^{\prime \prime}}, \theta_{p p^{\prime}}$ are the angles between $\mathbf{p}^{\prime}, \mathbf{p}^{\prime \prime}$ and $\mathbf{p}, \mathbf{p}^{\prime}$ respectively.

The values $\zeta_{p^{\prime} p^{\prime \prime}}$ and $\zeta_{p p^{\prime}}$ should be greater than or equal to zero, in order that three-phonon processes be allowed by the conservation laws (9) and (10). Having put quantities $\zeta_{p^{\prime} p^{\prime \prime}}$ and $\zeta_{p p^{\prime}}$ equal to zero, we obtain the boundaries of the regions in which threephonon processes can take place.

Substituting (3) into (12) and equating the resulting expression to zero, we obtain an equation in the fourth power of $p^{\prime}(p)$. Its solutions are

$$
{p^{\prime}}_{ \pm}(p)=\frac{1}{2}\left(p \pm \sqrt{3} \sqrt{p_{\text {max }}^{2}-p^{2}}\right), \quad p^{\prime}(p)=p,(13
$$

where

$$
p_{\max }=\sqrt{\frac{4}{5}} p_{c}
$$


Only three solutions of the quartic equation are written because the solution $p^{\prime}(p)=p$ is a 2 -fold root.

Having equated expression (11) to zero, after substituting relation (3) in it, we have an equation of the fourth power in $p^{\prime}\left(p^{\prime \prime}\right)$. The solutions of this equation are:

$p_{ \pm}^{\prime}\left(p^{\prime \prime}\right)=\frac{1}{2}\left(-p^{\prime \prime} \pm \sqrt{3} \sqrt{p_{\text {max }}^{2}-p^{\prime \prime}}\right), \quad p^{\prime}\left(p^{\prime \prime}\right)=-p^{\prime \prime}$.

Here the solution $p^{\prime}\left(p^{\prime \prime}\right)=-p^{\prime \prime}$ is also a 2-fold root. We note that the only physical solution in this case is $p_{+}^{\prime}\left(p^{\prime \prime}\right)$ because other solutions have one or both momenta with negative values.

Relations (13)-(15) determine the boundaries of regions where three-phonon processes are allowed. In Fig. 1, $a, b$, based on (13) and (15), the area where three-phonon processes are allowed is shown shaded. As we have used the dimensionless variable normalized to $p_{c}$, the specified area does not depend on pressure.

From relations (13)-(15) (see Fig. 1) it follows that values of the momentum of phonons participating in three-phonon processes, can change in the ranges:

$$
\begin{aligned}
0<\left(p^{\prime}, p^{\prime \prime}\right) & <\sqrt{\frac{3}{5}} p_{c}=p_{\min }, \\
0 & <p<p_{\max } .
\end{aligned}
$$

At saturated vapor pressure we have $\tilde{p}_{\min }=7.7 \mathrm{~K}$, and $\tilde{p}_{\max }=8.9 \mathrm{~K}$.

We note that $p_{\min }$ and $p_{\max }$ can be expressed in terms of the function $\psi$ and its first derivative. From the conservation laws (9) and (10) it follows that when $p^{\prime} \rightarrow p_{\min }, p^{\prime \prime} \rightarrow 0$. As a result of the fact that $p^{\prime \prime} \rightarrow 0$, from (9) we find, that $\mathbf{p}^{\prime}=\mathbf{p}$ and consequently $\zeta_{p p^{\prime}}=0$. Then, taking into account that $p \rightarrow p_{\min }+p^{\prime \prime}$, we expand expression (12) as a series in $p^{\prime \prime}$, with the result

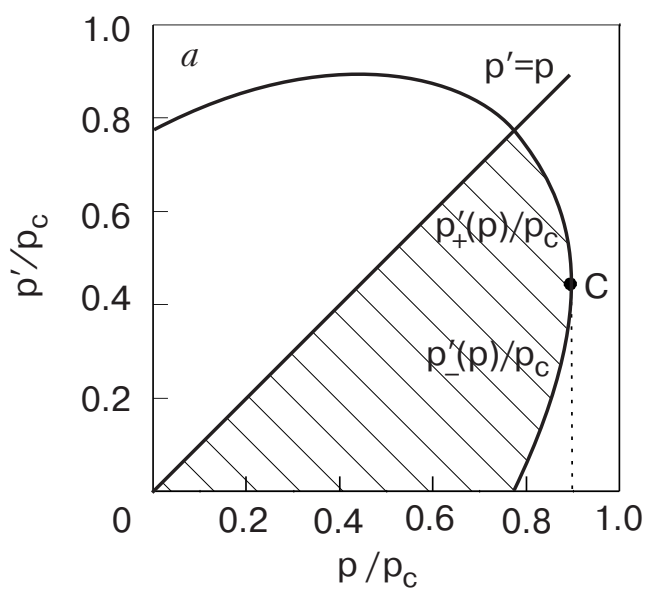

$$
\left.\{\psi+p d \psi / d p\}\right|_{p=p_{\min }}=0 .
$$

Taking into account expression (3), we have

$$
p_{\min }=\sqrt{\frac{3}{5}} p_{c}
$$

as obtained above.

The maximum value of the momentum of a phonon $p$ which is created as a result of combining phonons $p^{\prime}$ and $p^{\prime \prime}$, in accordance with [13], is defined by the equality

$$
\psi\left(p_{\max } / 2\right)=\psi\left(p_{\max }\right),
$$

from which, taking into account (3), we obtain relation (14).

Boundary values of the momenta of phonons participating in three-phonon processes were studied in Refs. 7,14-17, however, except Ref. 16, the regions of momenta where three-phonon processes are allowed (see Fig. 1), were not obtained. In Ref. 16, an area similar to that shown in Fig. 1, $a$ was shown. Nevertheless it has some difference with Fig. 1, $a$, apparently because a less adequate approximation to the phonon spectrum was used for the computer evaluations. In Refs. 7,14-17, analytical relations for the boundaries of the areas and the expression (18) for the boundary momentum of phonon $p^{\prime}$ expressed by means of the function $\psi$ and its first derivative were not obtained.

The probability density of three-phonon process, according to [18], can be written as

$$
W\left(\mathbf{p} \mid \mathbf{p}^{\prime} \mathbf{p}^{\prime \prime}\right)=\frac{\pi c}{\rho \hbar} p p^{\prime} p^{\prime \prime}(u+1)^{2} \delta_{\mathbf{p}^{\prime}+\mathbf{p}^{\prime \prime}, \mathbf{p}} .
$$

From (21) it is clear, that the probability density does not depend on the angles between the momenta of the interacting phonons as long as such processes

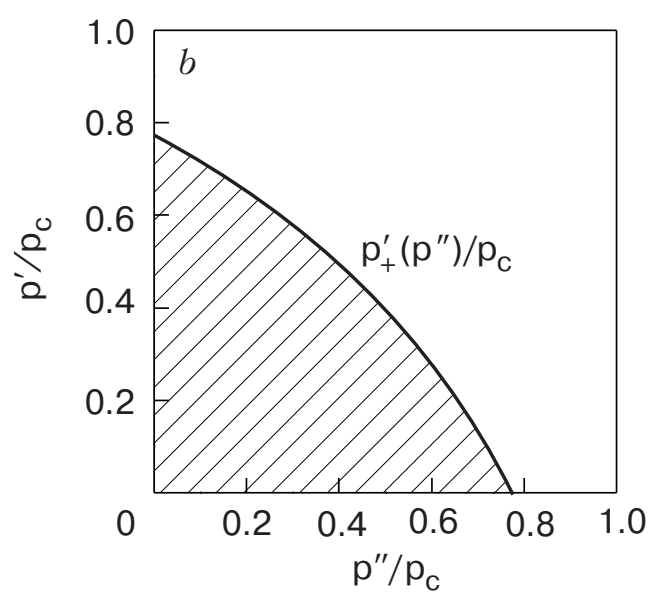

Fig. 1. The restrictions on the momenta of phonons participating in three-phonon processes. Three-phonon processes are allowed in the shaded areas. 
are allowed by the conservation laws (9) and (10). It follows from (21), that the most probable process is when

$$
\varepsilon^{\prime}=\varepsilon^{\prime \prime}=\varepsilon / 2
$$

Starting from relation (11), we calculate the angle between phonons with momenta $\mathbf{p}^{\prime}$ and $\mathbf{p}^{\prime \prime}$ as function of $p$ and the relation between the momenta of phonons $p^{\prime}$ and $p^{\prime \prime}$. For that we need the $p^{\prime}$ and $p^{\prime \prime}$ dependences on $p$ and $m=p^{\prime} / p^{\prime \prime}$ which, taking into account (10), can be written, in the zeroth approximation of $\psi$, as

$$
\begin{aligned}
& p^{\prime}=\frac{m}{m+1} p, \\
& p^{\prime \prime}=\frac{1}{m+1} p .
\end{aligned}
$$

Substituting (3) into (11), taking into account (23) and (24), and expanding the resulting expression $\zeta_{p^{\prime} p^{\prime \prime}}$ as a series in the small parameter $\theta_{p^{\prime} p^{\prime \prime}}$ up to second-order terms, we get

$$
\theta_{p^{\prime} p^{\prime \prime}}=\sqrt{8 \psi_{\text {max }} \frac{p^{2}}{p_{c}^{2}}\left[3-5 \frac{p^{2}}{p_{c}^{2}}\left(1-\frac{m}{(m+1)^{2}}\right)\right]} .
$$

From (25), it follows that when the value of $m$ is fixed, the function $\theta_{p^{\prime} p^{\prime \prime}}$ reaches the maximum value

$$
\theta_{p^{\prime} p^{\prime \prime}}\left(p=p_{\theta \max }\right)=3(m+1) \sqrt{\frac{2 \psi_{\max }}{5\left(m^{2}+m+1\right)}}
$$

at

$$
p_{\theta \max }=\sqrt{\frac{3}{10}} \frac{m+1}{\sqrt{m^{2}+m+1}} p_{c} .
$$

In accordance with (26), function $\theta_{p^{\prime}} p^{\prime \prime}$ reaches the maximum value at $m=1$, i.e., when the requirement (22) is satisfied. We note that at $m=1$

$$
p_{\theta \max }=\sqrt{\frac{2}{5}} p_{c}
$$

and

$$
\theta_{p^{\prime} p^{\prime \prime} \max }=\sqrt{\frac{24}{5} \psi_{\max }} .
$$

In Fig. 2 we show the dependence of the relation $\theta_{p^{\prime} p^{\prime \prime}} / \theta_{p^{\prime} p^{\prime \prime} \max }$ on $p / p_{c}$, for different relations between the momenta of phonons $p^{\prime}$ and $p^{\prime \prime}$ which we get from (25). Here $\theta_{p^{\prime} p^{\prime \prime} \max }$ is the maximum angle between the momenta of the phonons participating in the three-phonon process, which is defined by Eq. (29). We note that in the chosen variables, the form of this curve does not depend on pressure.

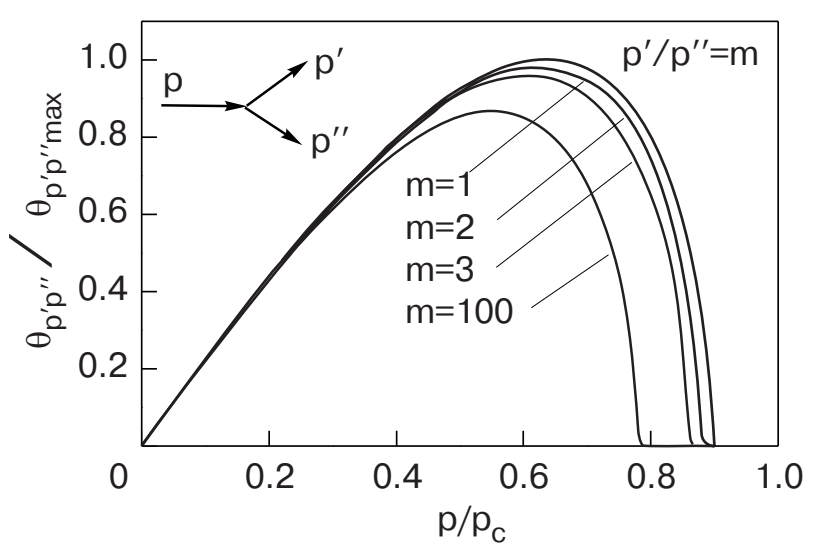

Fig. 2. The dependence of the angle $\theta_{p^{\prime}} p^{\prime \prime}$ between phonons with momenta $\mathbf{p}^{\prime}$ and $\mathbf{p}^{\prime \prime}$, on the momentum of the decay phonon $p$ for different ratios of the momenta of the created phonons $m=p^{\prime} / p^{\prime \prime}$.

In Table 1 , numerical values of angle $\theta_{p^{\prime}} p^{\prime \prime} \max$ and the critical momentum $\tilde{p}_{c}$ are given for different pressures which correspond to the conditions of the experiment [4].

Table 1. The pressure dependences of maximum angle $\theta_{p^{\prime} p^{\prime \prime} \max }$ (see Eq. (29)) between two phonons created in a $3 p p$ decay process and the critical momentum $\tilde{p}_{c}$ (see Eq. (6)).

\begin{tabular}{c|c|c}
\hline \hline$P$, bar & $\theta_{p^{\prime} p^{\prime \prime} \max }, \operatorname{deg}$ & $\tilde{p}_{C}, \mathrm{~K}$ \\
\hline \hline 0 & 27 & 10 \\
5 & 19 & 8.54 \\
10 & 12 & 6.24 \\
12 & 10 & 5.09 \\
18 & 5 & 0.83 \\
\hline \hline
\end{tabular}

It can be seen from Table 1 that the maximum angle of three-phonon processes decreases with increasing pressure. From (29) this can be explained as $\psi$ max decreasing with increasing pressure. Also when the value of pressure increases, the value of $p_{c}$, which determines the maximum values of the momenta of phonons which can participate in three-phonon interactions, decreases. These two factors lead to a reduction of the volume of momentum space in which three-phonon processes are allowed by the conservation laws of energy and momentum.

\section{The rates of three-phonon processes in isotro- pic phonon systems}

The rates of three phonon processes in isotropic phonon systems, at the saturated vapor pressure, were calculated in [18]. Experiments [4] were carried out 
at pressure of $0,5,12$ and 18 , so in this work we investigate the rates at different pressures. Besides this the expression obtained in Ref. 18 for the rate of three-phonon processes is valid only in range $0<p<p_{\min }$. Here we shall carry out a detailed analysis of the rate of three-phonon processes in the range of momenta $p_{\min }<p<p_{\max }$ in which the rate of three-phonon processes changes from the maximum value to zero. Some results of this section were reported on International Conference Phonons 2004 (see Ref. 19) and were partly published in [20].

We follow the scheme of the Ref. 18 to calculate the rate of three phonon processes, starting from the kinetic equation:

$$
\begin{gathered}
\frac{d n_{1}}{d t}=\frac{1}{2} \int \frac{W\left(\mathbf{p}_{1} \mid \mathbf{p}_{2} \mathbf{p}_{3}\right)}{(2 \pi \hbar)^{3}} \times \\
\times\left[n_{2} n_{3}\left(1+n_{1}\right)-n_{1}\left(1+n_{2}\right)\left(1+n_{3}\right)\right] \times
\end{gathered}
$$

$\times \delta\left[\varepsilon\left(\mathbf{p}_{1}\right)-\varepsilon\left(\mathbf{p}_{2}\right)-\varepsilon\left(\mathbf{p}_{3}\right)\right] \delta\left(\mathbf{p}_{1}-\mathbf{p}_{2}-\mathbf{p}_{3}\right) d^{3} p_{2} d^{3} p_{3}+$ $+\int \frac{W\left(\mathbf{p}_{3} \mid \mathbf{p}_{1} \mathbf{p}_{2}\right)}{(2 \pi \hbar)^{3}}\left[n_{3}\left(1+n_{1}\right)\left(1+n_{2}\right)-n_{1} n_{2}\left(1+n_{3}\right)\right] \times$ $\times \delta\left[\varepsilon\left(\mathbf{p}_{3}\right)-\varepsilon\left(\mathbf{p}_{1}\right)-\varepsilon\left(\mathbf{p}_{2}\right)\right] \delta\left(\mathbf{p}_{3}-\mathbf{p}_{1}-\mathbf{p}_{2}\right) d^{3} p_{2} d^{3} p_{3}$,

where $n_{i}=n\left(\mathbf{p}_{i}\right)$ is the number of phonons in the given quantum state, and $W\left(\mathbf{p}_{3} \mid \mathbf{p}_{1} \mathbf{p}_{2}\right)$ is the probability density of transitions in phase space, which is defined by expression (21).

To calculate the typical rate of three-phonon processes in isotropic phonon systems, we follow [18], and substitute in the Eq. (30):

$$
n_{1}=n_{10}+\delta n, \quad n_{2}=n_{20}, \quad n_{3}=n_{30},
$$

where the subscript $\ll 0 »$ specifies an equilibrium distribution

$$
n_{0}(p)=\left\{\exp \left[\varepsilon(p) / k_{B} T\right]-1\right\}^{-1},
$$

and $\delta n$ is the deviation of the distribution function from equilibrium.

The typical rate of three-phonon processes $v_{3 p p}$ is naturally defined by the equation

$$
v_{3 p p}\left(\mathbf{p}_{1}\right)=-\frac{1}{\delta n} \frac{d \delta n}{d t} .
$$

Starting from expressions (21), (30)-(33) we have

$$
v_{3 p p}\left(\mathbf{p}_{1}\right)=\frac{(u+1)^{2}}{4 \pi \rho \hbar^{4}}\left\{\frac{1}{2} J_{1}\left(\mathbf{p}_{1}\right)+J_{2}\left(\mathbf{p}_{1}\right)\right\},
$$

where

$$
\begin{gathered}
J_{1}\left(\mathbf{p}_{1}\right)=\frac{c p_{1}}{n_{10}} \times \\
\times \int_{0}^{p_{1}} d p_{2} \int_{0}^{2} d \zeta_{12} p_{2}^{3}\left|\mathbf{p}_{1}-\mathbf{p}_{2}\right| n_{0}\left(p_{2}\right) n_{0}\left(p_{1}-p_{2}\right) \times \\
\times \delta\left(\varepsilon\left(p_{1}\right)-\varepsilon\left(p_{2}\right)-\varepsilon\left(\left|\mathbf{p}_{1}-\mathbf{p}_{2}\right|\right)\right), \\
J_{2}\left(\mathbf{p}_{1}\right)=c p_{1} \times \\
\times \int_{p_{1}}^{p_{\max }} d p_{3} \int_{0}^{2} d \zeta_{13} p_{3}^{3}\left|\mathbf{p}_{3}-\mathbf{p}_{1}\right|\left\{n_{0}\left(p_{3}-p_{1}\right)-n_{0}\left(p_{3}\right)\right\} \times \\
\times \delta\left[\varepsilon\left(p_{3}\right)-\varepsilon\left(p_{1}\right)-\varepsilon\left(\left|\mathbf{p}_{3}-\mathbf{p}_{1}\right|\right)\right] .
\end{gathered}
$$

Here

$$
\begin{aligned}
& \zeta_{12}=1-\frac{\mathbf{p}_{1} \mathbf{p}_{2}}{p_{1} p_{2}}, \\
& \zeta_{13}=1-\frac{\mathbf{p}_{1} \mathbf{p}_{3}}{p_{1} p_{3}} .
\end{aligned}
$$

Doing the integration on $\zeta_{12}$ and $\zeta_{13}$ with the help of the $\delta$-functions in Eqs. (35) and (36), we obtain for $v_{3 p p}\left(\mathbf{p}_{1}\right)$ from (34) the following relation:

$$
v_{3 p p}\left(\tilde{p}_{1}\right)=\frac{(u+1)^{2}}{4 \pi \rho \hbar^{4}}\left(\frac{k_{B}}{c}\right)^{5}\left\{\frac{1}{2} \xi\left(\tilde{p}_{1}\right)+\beta\left(\tilde{p}_{1}\right)\right\},
$$

where $\tilde{p}_{i}=c p_{i} / k_{B}$,

$\xi\left(\tilde{p}_{1}\right)=\int_{0}^{\tilde{p}_{1}} d \tilde{p}_{2} \tilde{p}_{2}^{2}\left(\tilde{p}_{1}-\tilde{p}_{2}\right)^{2}\left(1+2 \tilde{n}_{0}\left(\tilde{p}_{2}\right)\right) \eta\left(\zeta\left(\tilde{p}_{1}, \tilde{p}_{2}\right)\right)$

$$
\begin{gathered}
\beta\left(\tilde{p}_{1}\right)=\int_{\tilde{p}_{1}}^{\tilde{p}_{\max }} d \tilde{p}_{3} \tilde{p}_{3}^{2}\left(\tilde{p}_{3}-\tilde{p}_{1}\right)^{2} \times \\
\times\left\{\tilde{n}_{0}\left(\tilde{p}_{3}-\tilde{p}_{1}\right)-\tilde{n}_{0}\left(\tilde{p}_{3}\right)\right\} \eta\left(\zeta\left(\tilde{p}_{3}, \tilde{p}_{1}\right)\right) .
\end{gathered}
$$

Here

$$
\begin{gathered}
\tilde{n}_{0}(\tilde{p})=[\exp (\tilde{p} / T)-1]^{-1}, \\
\zeta\left(\tilde{p}_{i}, \tilde{p}_{j}\right)=\frac{\tilde{p}_{i}-\tilde{p}_{j}}{\tilde{p}_{i} \tilde{p}_{j}} \times \\
\times\left(\tilde{p}_{i} \psi\left(k_{B} \tilde{p}_{i} / c\right)-\tilde{p}_{j} \psi\left(k_{B} \tilde{p}_{j} / c\right)-\right. \\
\left.-\left(\tilde{p}_{i}-\tilde{p}_{j}\right) \psi\left(k_{B}\left(\tilde{p}_{i}-\tilde{p}_{j}\right) / c\right)\right)
\end{gathered}
$$

and

$$
\eta(x)=\left\{\begin{array}{l}
1, x \geq 0 \\
0, x<0 .
\end{array}\right.
$$

Expression (39), together with (40)-(44), completely determines the typical rate of three-phonon relaxation in isotropic phonon systems. We underline 
the fact that the final result (39) contains parameters that describe the nonlinearity of the phonon spectrum of He II. Mathematically this fact is expressed by the appearance of $\eta$-functions which restrict the integration range. The rates represented in [18] do not contain these $\eta$ functions. We note that these $\eta$ functions cause the rapid decrease of the rates near the boundary momentum $p_{\max }$.

Starting from expression (39), we get an analytical expression for rate of a three-phonon scattering in the case $p_{\min }<p_{1}<p_{\max }$. When $p_{1}>p_{\min }$ the term $\beta\left(\tilde{p}_{1}\right)$ is equal to zero because phonons with momentum $p_{1}>p_{\text {min }}$ cannot participate in combining processes (see Fig. 1,b). In this case expression (39) will have only the first term $\xi\left(\tilde{p}_{1}\right)$, which is defined by expression (40). It corresponds to the decay of a phonon with momentum $p_{1}$. We note that the term $\xi\left(\tilde{p}_{1}\right)$ describes the stimulated decay of a phonon with momentum $p_{1}$ which corresponds to the term with the Bose function, in the brackets of the integrand of (40), and spontaneous decay of a phonon with momentum $p_{1}$ which corresponds to the first term, unity, in the brackets. In our case $p_{\min }<p_{1}<p_{\max }$ we do not take into account stimulated decay, because its rate is much smaller than the rate of spontaneous decay. If the momentum of one of the phonons, which is created as a result of decay, is small, then the contribution of such decay processes in expression (40) is small, too. Also when the momenta of both phonons are significant, the term with the Bose function is much smaller than unity. As a result, we can neglect the term that contains the Bose function in the brackets of the integrand (40). On integrating we have:

$$
\begin{gathered}
v_{3 p p}=\frac{(u+1)^{2}}{240 \pi \rho \hbar^{4}} \sqrt{3} \sqrt{p_{\max }^{2}-p_{1}^{2}} \times \\
\times\left(9 p_{1}^{4}-14 p_{1}^{2} p_{\min }^{2}+6 p_{\min }^{4}\right) .
\end{gathered}
$$

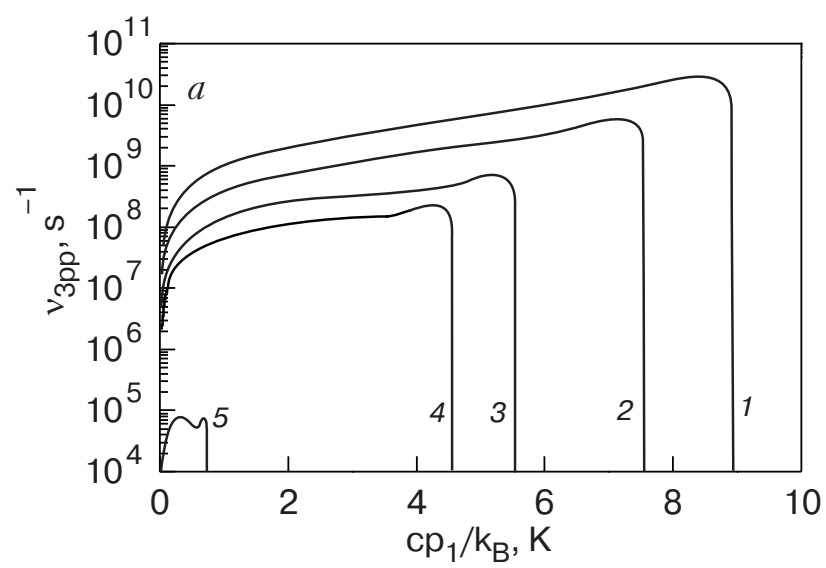

Expression (45), in the range $p_{1} \rightarrow p_{\max }$, decreases rapidly and goes to zero at $p_{1}=p_{\max }$ due to the radicand going to zero. The expression in the brackets increases but tends to a finite value equal to $1.2 p_{c}^{4}$. As a result, the rate decreases from a maximum to zero in a small range of momenta (see Fig. 3 ). The change of the rate is determined by a competition between the expression under square root, which decreases as $p_{1} \rightarrow p_{\max }$, and the factor in the brackets. As a result of the competition, expression (45) reaches a maximum value at $p_{1} \approx 0.84 p_{c}$, and at $p_{1} \approx 0.89 p_{c}$ it is equal to zero. Therefore the rate changes from the maximum value to zero in a small momentum range.

We now consider the typical time of three-phonon processes. Equation (39) can be interpreted as the reciprocal lifetime of a phonon with momentum $p_{1}$ in an equilibrium field of thermal phonons with temperature $T$. Let us consider the limiting cases which follow from Eq. (39). First, we consider the hypersound limit, when $\tilde{p}_{1} / T>1$. Thus

$$
\beta\left(\tilde{p}_{1}\right) \approx 0
$$

and

$$
\xi\left(\tilde{p}_{1}\right) \approx \int_{0}^{\tilde{p}_{1}} d \tilde{p}_{2} \tilde{p}_{2}^{2}\left(\tilde{p}_{1}-\tilde{p}_{2}\right)^{2}=\frac{\tilde{p}_{1}^{5}}{30} .
$$

Substitution of relations (46) and (47) in (39) gives

$$
v_{3 p p}^{(\infty)}\left(p_{1}\right)=\frac{(u+1)^{2}}{240 \pi \rho \hbar^{4}} p_{1}^{5} .
$$

This expression was obtained in Ref. 21 for the first time. From relations (46) and (47) it is clear that this limiting case corresponds only to decay processes as $\mathbf{p}_{1} \rightarrow \mathbf{p}_{2}+\mathbf{p}_{3}$. It is should be noted, that formula (48) has actually a rather restricted area of application. This is because two requirements must be satis-

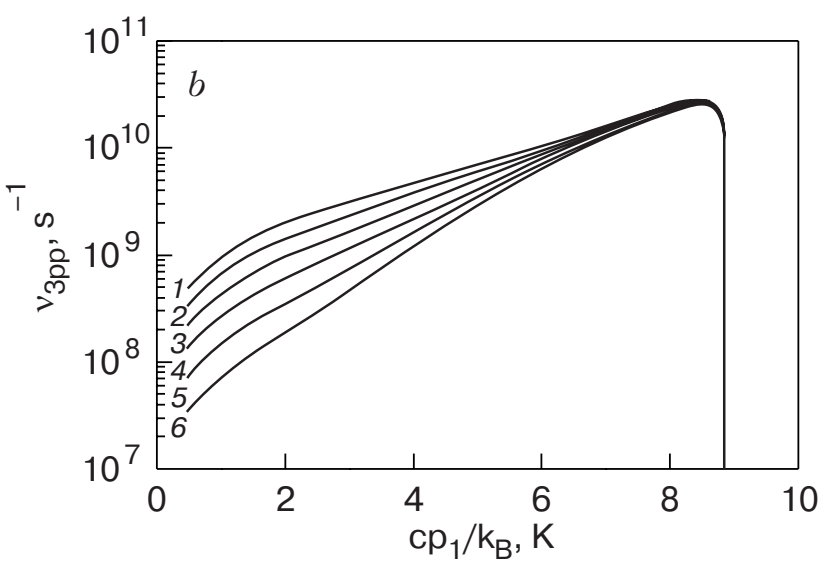

Fig. 3. The dependence of the relaxation rate in isotropic phonon systems $v_{3 p p}$ on momentum $p_{1}$. In Fig. 3, $a$ curves $1-5$ are for pressures $0,5,10,12,18$ bar respectively, at $T=1 \mathrm{~K}$. In Fig. 3,b curves $1-6$ are for temperatures 1, 0.9, 0.8, 0.7, $0.6,0.5 \mathrm{~K}$ respectively, at the saturated vapor pressure. 
fied: $\tilde{p}_{1} / T>>1$ and $\tilde{p}_{1}<\tilde{p}_{\min }$; therefore it can be used for numerical evaluations for pressures up to 12 bar at temperatures up to $1 \mathrm{~K}$, and at lower pressures if the temperature is higher.

The other limiting case is $\tilde{p}_{1} / T<<1$, corresponding to the absorption of acoustic sound waves by an equilibrium phonon field with temperature $T$. In this case

$$
\xi\left(\tilde{p}_{1}\right) \approx 0
$$

and

$$
\beta\left(\tilde{p}_{1}\right) \approx \frac{\tilde{p}_{1}}{T} \int_{0}^{\infty} \frac{\tilde{p}_{3}^{4} \exp \left(\tilde{p}_{3} / \mathrm{T}\right)}{\left(\exp \left(\tilde{p}_{3} / \mathrm{T}\right)-1\right)^{2}} d \tilde{p}_{3}=T^{4} \frac{4 \pi^{4}}{15} \tilde{p}_{1} .
$$

Substituting (49) and (50) into (39), we have

$$
v_{3 p p}^{(0)}\left(p_{1}\right)=\frac{\pi^{3}(u+1)^{2}}{15 \rho \hbar^{4}}\left(\frac{k_{B}}{c}\right)^{4} T^{4} p_{1},
$$

and thus we come to the result obtained in Refs. 22,23 . We note that in expression (50) we replace $\tilde{p}_{\max }$ by $\infty$. Such a replacement can be made when $\tilde{p}_{\max } / T \gg>1$. There was no such requirement in [18] because the condition is always satisfied at the saturated vapor pressure. However with increasing of pressure, it starts to play an important role and restricts the area of applicability of expression (51).

In contrast to the process of decay of high-energy phonons (see (48)), mentioned above, the limiting case (51), which follows from relations (49), (50), corresponds only to combining processes such as $\mathbf{p}_{1}+\mathbf{p}_{2} \rightarrow \mathbf{p}_{3}$. Equation (51) has been used many times for the description of various relaxation processes in pure He II and also in superfluid mixtures of ${ }^{3} \mathrm{He}^{-}{ }^{4} \mathrm{He}$ (see Ref. 24).

It is important to note that equation (39), taking into account dependences (3)-(8), is valid for any pressure at which the dispersion is anomalous. In Fig. 3, rates for three-phonon processes are shown for different pressures and temperatures. In Fig. 3, $a$ it can be seen that with increasing of pressure, the rate begins to decrease. This is caused by the decreasing of value $p_{c}$, and by the change in the parameters $u, \rho$, and $c$ of superfluid helium. As pressure increases from 0 up to 12 , at fixed value of $p_{c}$, the rates decrease $\approx$ five times, and as the pressure is further increased up to 18 , the rate decreases approximately 2 times. Another cause of decreasing rates with increasing pressure is the decreasing momentum range in which three-phonon processes are allowed. This is due to the decreasing of value $p_{c}$ which leads to a reduction of the phase volume in the integration.

We note that the decreasing rates, which is connected with the decreasing of value $p_{c}$, has a strong temperature dependence: at temperatures $T \sim 1 \mathrm{~K}$, the decreasing of value $p_{c}$ with growth of pressure from 0 to $12 \mathrm{bar}$, at fixed parameters $u, \rho$ and, $c$, leads to the rates decreasing up to 2 times, and for a further increase of pressure to $18 \mathrm{bar}$, the rate decreases up to 300 times. At temperatures $T<0.1 \mathrm{~K}$, the decreasing of value $p_{c}$ with growth of pressure from 0 up to 18 bar does not affect the rates of three-phonon relaxation which in this case is caused only by the change in the parameters of liquid helium. Such a temperature dependence can be explained by presence of the Bose-Einstein functions which contain the expression $\exp (\tilde{p} / T)$ in the integrands.

The temperature dependence of the rate, $v_{3 p p}$, in the limiting cases (48) and (51), as one can see in Fig. $3, b$, is as follows: at small values of $p_{1}$, $v_{3 p p} \propto T^{4}$, and at large values of $p_{1}, v_{3 p p}$ has practically no temperature dependence.

\section{Phonon distribution function of an anisotropic phonon system}

Systems with an anisotropic distribution of phonons in momentum space are created in experiments (see, for example, Refs. 1-5) using a heater immersed in superfluid helium ${ }^{4} \mathrm{He}$, which is at such a low temperature that the effect of thermal excitations can be neglected. The heater is a metal film evaporated onto glass. When current flows through the metal film, phonons are created in the superfluid helium within a narrow cone with a solid angle $\Omega_{p}<1$ and with an axis perpendicular to the surface of the heater. The dimensions in coordinate space of this strongly anisotropic phonon system are defined by the area of the heater and the duration of the thermal pulse.

Such anisotropic phonon systems can be described with a help of approximate quasi-equilibrium distribution function (see, for example, Refs. 25-28) which can be written as

$$
n_{p}(\mathbf{p})=\eta\left(\theta_{p}-\theta\right)\left[\exp \left(\varepsilon / k_{B} T_{p}\right)-1\right]^{-1} .
$$

This distribution function has simple physical meaning and includes all the necessary parameters of anisotropic phonon systems: temperature $T_{p}$, and the value of an anisotropy which is given by angle $\theta_{p}$. The approximation (52) has allowed the successful solution of a number of problems [20,25-28].

However, this function does not make the integral of the three-phonon collisions equal to zero. The exact equilibrium distribution function of phonons in anisotropic phonon systems, in accordance with Ref. 29, is 


$$
n_{u}(\mathbf{p})=\left\{\exp \left(\frac{\varepsilon-\mathbf{p u}}{k_{B} T}\right)-1\right\}^{-1}
$$

where

$$
\mathbf{u}=\mathbf{N} c(1-\chi),
$$

is the drift velocity, $\mathbf{N}$ is the unit vector directed along the full momentum of phonon system, which defines the axis of anisotropy of phonon system, and $\chi$ is the parameter of anisotropy. It is obvious that function (53) makes the integral of the three-phonon collisions equal to zero. In order to derive the further results we use exact equilibrium distribution function (53).

In a weakly anisotropic case the parameter $\chi$ is close to unity. In our case, which corresponds to the experiments [4,5], the phonon pulses are strongly anisotropic phonon systems and $\chi<<1$.

The expression (53), taking into account (1) and (54), can be written as

$$
n_{u}(p, \zeta)=\left\{\exp \left(\frac{c p}{k_{B} T}(\psi+\chi+\zeta-\zeta \chi)\right)-1\right\}^{-1}
$$

where $\zeta=1-\mathbf{p N} / p$.

The values of the parameter of anisotropy $\chi$ and temperature $T$, which are contained in the distribution function (55), can be related to the values of the parameters $\theta_{p}$ and $T_{p}$ of the approximate distribution functions (52), which have a clear physical meaning. Starting from the equality of energies and momenta of anisotropic phonon systems, calculated with a help of distribution functions (52) and (55), we have a system of two equations which connects parameters $\theta_{p}$ and $T_{p}$ with parameters $\chi$ and $T$ :

$$
\begin{aligned}
& \int \varepsilon n_{p} d^{3} p=\int \varepsilon n_{u} d^{3} p, \\
& \int \mathbf{p} n_{p} d^{3} p=\int \mathbf{p} n_{u} d^{3} p .
\end{aligned}
$$

This system can be solved numerically relating parameters $\chi$ and $T$, to values of parameters $\zeta_{p}=1-\cos \theta_{p}$ and $T_{p}$. In the following calculations we always use the relation

$$
\zeta_{p}=\psi_{\max } / 2
$$

which is a good approximation to the real situation and represents a pressure dependence of $\zeta_{p}$. Values of parameters $\chi$ and $T$ which correspond to parameters $\zeta_{p}$ and $T_{p}$ can be obtained solving the combined equations (56),(57). These values will be used in our further calculations and are represented in Table 2. We note that the choice of pressures in this paper corresponds to the choice of pressures in experiments [4].
Table 2 . The relation between parameters $\zeta_{p}, T_{p}$ of the ap-

\begin{tabular}{|c|c|c|c|c|c|c|}
\hline $\begin{array}{l}P, \\
\text { bar }\end{array}$ & $\zeta_{p}$ & $\begin{array}{c}T_{p} \\
\mathrm{~K}\end{array}$ & $\chi$ & $\begin{array}{c}T, \\
\mathrm{~K} \\
\end{array}$ & $\begin{array}{c}\langle\tilde{p}\rangle, \\
\mathrm{K}\end{array}$ & $\begin{array}{c}\theta_{\text {eff }}, \\
\text { deg }\end{array}$ \\
\hline \multirow{6}{*}{0} & \multirow{6}{*}{0.023} & 0.5 & 0.019 & 0.016 & 0.934 & 11 \\
\hline & & 0.6 & 0.020 & 0.021 & 1.075 & 13 \\
\hline & & 0.7 & 0.019 & 0.025 & 1.207 & 14 \\
\hline & & 0.8 & 0.020 & 0.030 & 1.317 & 15 \\
\hline & & 0.9 & 0.020 & 0.036 & 1.462 & 17 \\
\hline & & 1 & 0.020 & 0.041 & 1.581 & 18 \\
\hline \multirow{6}{*}{5} & \multirow{6}{*}{0.011} & 0.5 & 0.0093 & 0.0084 & 0.906 & 9 \\
\hline & & 0.6 & 0.013 & 0.012 & 0.992 & 10 \\
\hline & & 0.7 & 0.010 & 0.014 & 1.183 & 11 \\
\hline & & 0.8 & 0.010 & 0.016 & 1.28 & 12 \\
\hline & & 0.9 & 0.010 & 0.019 & 1.424 & 13 \\
\hline & & 1 & 0.010 & 0.022 & 1.569 & 14 \\
\hline \multirow{6}{*}{10} & \multirow{6}{*}{0.0046} & 0.5 & 0.0043 & 0.0041 & 0.835 & 7 \\
\hline & & 0.6 & 0.0044 & 0.0053 & 0.964 & 8 \\
\hline & & 0.7 & 0.0045 & 0.0064 & 1.085 & 9 \\
\hline & & 0.8 & 0.0044 & 0.0076 & 1.232 & 10 \\
\hline & & 0.9 & 0.0043 & 0.0086 & 1.361 & 10 \\
\hline & & 1 & 0.0041 & 0.0095 & 1.492 & 11 \\
\hline \multirow{6}{*}{12} & \multirow{6}{*}{0.003} & 0.5 & 0.0029 & 0.0029 & 0.788 & 6 \\
\hline & & 0.6 & 0.0029 & 0.0037 & 0.919 & 7 \\
\hline & & 0.7 & 0.0028 & 0.0044 & 1.074 & 8 \\
\hline & & 0.8 & 0.0027 & 0.0050 & 1.208 & 9 \\
\hline & & 0.9 & 0.0025 & 0.0056 & 1.349 & 9 \\
\hline & & 1 & 0.0024 & 0.0060 & 1.442 & 10 \\
\hline \multirow{6}{*}{18} & \multirow{6}{*}{0.00079} & 0.5 & 0.00018 & 0.00057 & 0.368 & 5 \\
\hline & & 0.6 & 0.000092 & 0.00062 & 0.403 & 5 \\
\hline & & 0.7 & 0.000037 & 0.00066 & 0.429 & 5 \\
\hline & & 0.8 & 0.000011 & 0.00071 & 0.455 & 4 \\
\hline & & 0.9 & 0.0000034 & 0.00078 & 0.484 & 4 \\
\hline & & 1 & 0.0000022 & 0.00084 & 0.506 & 3 \\
\hline
\end{tabular}
proximate distribution function (51) and the parameters $\chi$, $T$ of the local equilibrium distribution function (54) at different pressures. The dependence of the average momentum $\langle\tilde{p}\rangle$ on parameters $\chi, T$ and the angle $\theta_{\text {eff }}$ at which two phonons combine with momenta $\langle\tilde{p}\rangle$.

The value $\langle\tilde{p}\rangle$ given in Table 2 corresponds to the average momentum of phonons in a pulse and is determined by expression 


$$
\langle\tilde{p}\rangle=\frac{\int \tilde{p} n_{u} d^{3} p}{\int n_{u} d^{3} p}
$$

and $\theta_{\text {eff }}$ is an angle between the two interacting phonons with momenta $\langle\tilde{p}\rangle$ which is defined by conservation laws of energy and momentum.

\section{Relaxation in anisotropic phonon systems, caused by three-phonon processes}

The expression for the rate of three-phonon processes in anisotropic phonon systems, can be obtained from the kinetic equation (30) in a way similar to obtaining expression (39) for the rate of three-phonon processes in isotropic phonon systems:

$$
v_{3 p p}\left(\tilde{p}_{1}, \zeta_{1}\right)=\frac{(u+1)^{2}}{4 \pi \rho \hbar^{4}}\left(\frac{k_{B}}{c}\right)^{5} \times\left\{\frac{1}{2} \xi_{a}\left(\tilde{p}_{1}, \zeta_{1}\right)+\beta_{a}\left(\tilde{p}_{1}, \zeta_{1}\right)\right\}
$$

where

$$
\begin{gathered}
\xi_{a}\left(\tilde{p}_{1}, \zeta_{1}\right)=\int_{0}^{\tilde{p}_{1}} d \tilde{p}_{2} \tilde{p}_{2}^{2}\left(\tilde{p}_{1}-\tilde{p}_{2}\right)^{2} \eta\left(\zeta\left(\tilde{p}_{1}, \tilde{p}_{2}\right)\right)\left(1+\frac{2}{\pi} \int_{\zeta_{1,2-}}^{\zeta_{1,2+}} d \zeta_{2} n_{u}\left(k_{B} \tilde{p}_{2} / c, \zeta_{2}\right)\left\{\left(\zeta_{1,2+}-\zeta_{2}\right)\left(\zeta_{2}-\zeta_{1,2-}\right)\right\}^{-1 / 2}\right), \\
\beta_{a}\left(\tilde{p}_{1}, \zeta_{1}\right)=\int_{\tilde{p}_{1}}^{\tilde{p}_{\max }} d \tilde{p}_{3} \tilde{p}_{3}^{2}\left(\tilde{p}_{3}-\tilde{p}_{1}\right)^{2} \eta\left(\zeta\left(\tilde{p}_{3}, \tilde{p}_{1}\right)\right)\left(\beta_{2}-\beta_{3}\right) .
\end{gathered}
$$

Here $\zeta_{i}=1-\frac{\mathbf{p}_{i} \mathbf{N}}{p_{i}}$,

$$
\begin{gathered}
\beta_{2}=\frac{1}{\pi} \int_{\zeta_{1,(3-1)-}}^{\zeta_{1,(3-1)+}} d \zeta_{2} \frac{n_{u}\left(k_{B}\left(\tilde{p}_{3}-\tilde{p}_{1}\right) / c, \zeta_{2}\right)}{\sqrt{\left(\zeta_{1,(3-1)+}-\zeta_{2}\right)\left(\zeta_{2}-\zeta_{1,(3-1)-}\right)}}, \\
\beta_{3}=\frac{1}{\pi} \int_{\zeta_{3,1-}}^{\zeta_{3,1+}} d \zeta_{3} \frac{n_{u}\left(k_{B} \tilde{p}_{3} / c, \zeta_{3}\right)}{\sqrt{\left(\zeta_{3,1+}-\zeta_{3}\right)\left(\zeta_{3}-\zeta_{3,1-}\right)}}, \\
\zeta_{i, j \pm}=\zeta_{1}+\zeta\left(\tilde{p}_{i}, \tilde{p}_{j}\right)-\zeta_{1} \zeta\left(\tilde{p}_{i}, \tilde{p}_{j}\right) \pm \sqrt{\left(2 \zeta_{1}-\zeta_{1}^{2}\right)\left[2 \zeta\left(\tilde{p}_{i}, \tilde{p}_{j}\right)-\zeta^{2}\left(\tilde{p}_{i}, \tilde{p}_{j}\right)\right]},
\end{gathered}
$$

and $\zeta\left(\tilde{p}_{i}, \tilde{p}_{j}\right)$ is defined by relation (43).

As in the isotropic case, the first term in expression (60) corresponds to the decay of a phonon with momentum $\mathbf{p}_{1}$, and the second to the combining of a phonon with momentum $\mathbf{p}_{1}$ with phonons of the anisotropic phonon system. The relation for the rate of three-phonon processes in the anisotropic case differs from the isotropic case only by the dependence of phonon distribution function on the angle $\zeta$. As a consequence, the integration cannot now be made analytically. We note that if we substitute the distribution function (55) with the distribution function of phonons in the isotropic phonon system (32) in expression (60), then the integration over angles can be made and expression (60) for the rates of three-phonon processes in anisotropic phonon systems will turn into expression (39).

Figures can be obtained with a help of expression (60). Figure 4, $a$ shows dependence of the relaxation rate of a phonon with momentum $\mathbf{p}_{1}$ in anisotropic phonon systems on the angle $\theta_{1}$ between the axis of anisotropy of phonon system $\mathbf{N}$ and phonon momen- tum $\mathbf{p}_{1}$ at $T_{p}=1 \mathrm{~K}$ and different pressures. Values of $\zeta_{p}$ corresponding to different pressures is calculated with the help of equality (58) (see Table 2). The phonon momentum $p_{1}$ is considered to be equal to the average momentum of phonons in a pulse $\langle p\rangle$. It follows from Fig. 4, $a$, that the rates of three-phonon processes decrease with increasing pressure. This has the same cause as for the isotropic case. We note that with the growth of angle $\theta_{1}$ the rate $v_{3 p p}$ increases, reaching a maximum value, and then decreases and ceases to depend on an angle. In order to understand such angular dependence of the rates we turn to Fig. 5. In Fig. 5, curve $1+2$ is the same as curve 1 in Fig. 4, $a$. It is the sum of curves 1 and 2 , which correspond to the first and the second terms of expression (60), respectively. Curve 1 in Fig. 5 corresponds to the decay of a phonon with momentum $\mathbf{p}_{1}$, and curve 2 , to the combining of a phonon with momentum $\mathbf{p}_{1}$ with a phonon of the anisotropic phonon system. The increase in the rates, which is represented by curve 1 at small values of angle, is caused by stimulated decay of a phonon with momentum $\mathbf{p}_{1}$ due to the presence of the 

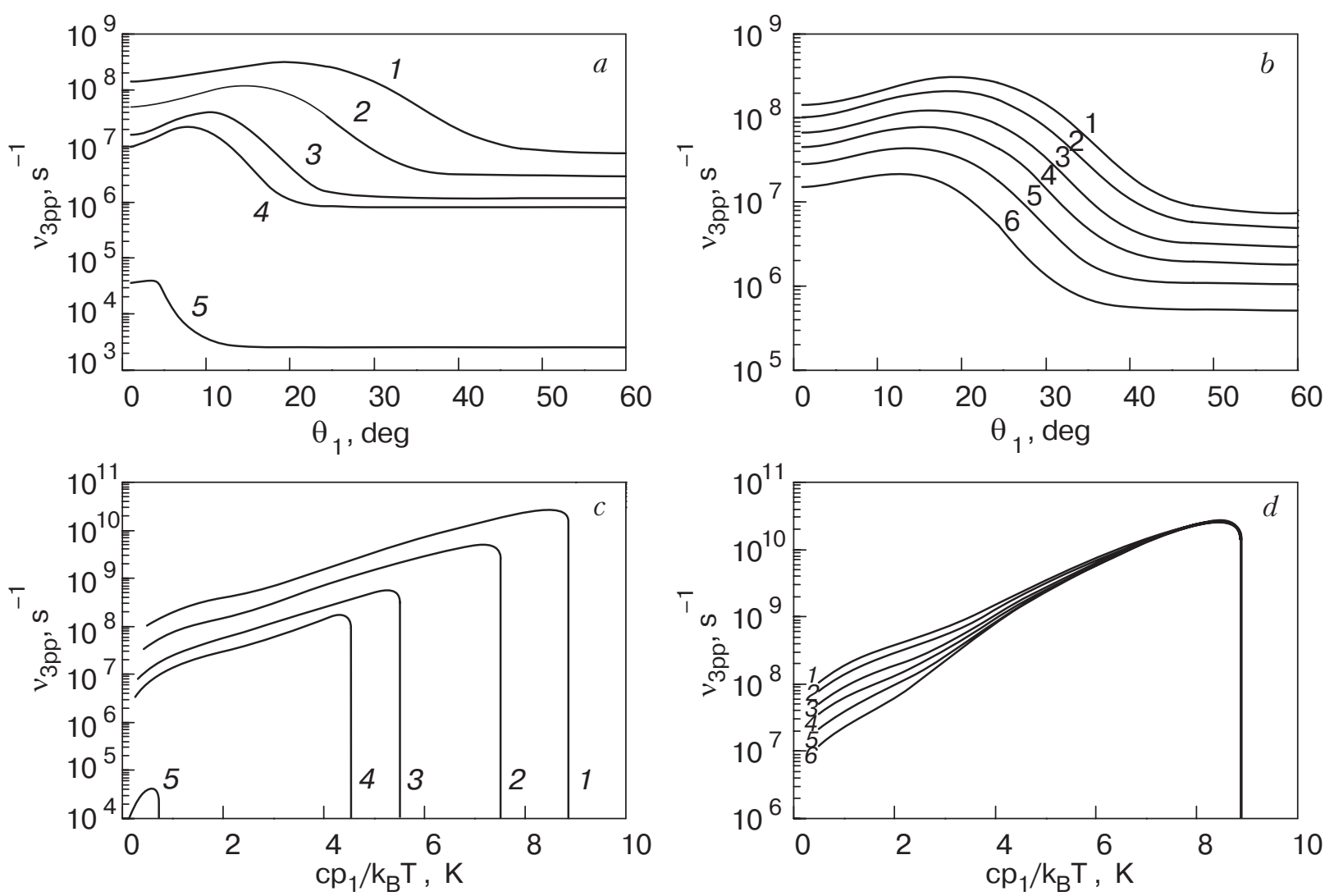

Fig. 4. The relaxation rates $v_{3 p p}$ in anisotropic phonon systems. Figure $4, a$ represents the dependence of $v_{3 p p}$ on $\theta_{1}$ at different pressures. Curves $1-5$ are for pressures $0,5,10,12,18$ bar, respectively, at $T_{p}=1 \mathrm{~K}$ and values of $\zeta_{p}$, which were defined from Eq. (57) (see Table 2). The momentum $\tilde{p}_{1}$ was considered to be equal to the average value of momentum $\langle\tilde{p}\rangle$ of the phonons in a pulse. Figure $4, b$ shows the dependence of $v_{3 p p}$ on $\theta_{1}$ at different temperatures. Curves $1-6$ are for temperatures $1,0.9,0.8,0.7,0.6,0.5 \mathrm{~K}$, respectively, at the saturated vapor pressure and when $\tilde{p}_{1}=\langle\tilde{p}\rangle($ see Table 2$)$ at $\zeta_{p}=0.023$. Figures $4, c, d$ show the $v_{3 p p}$ dependence on $\tilde{p}_{1}$, at the angle which corresponds to the maximum of curves in Fig. 4, $a$ and 4,b. The value of $\zeta_{p}$ was defined from equality (57). Figure 4,c shows the effect of different pressures $(0,5$, $10,12,18 \mathrm{bar})$ at $T_{p}=1 \mathrm{~K}$ and Fig. $4, d$ shows the effect of different temperatures $(1,0.9,0.8,0.7,0.6,0.5 \mathrm{~K})$ at the saturated vapor pressure $(d)$.

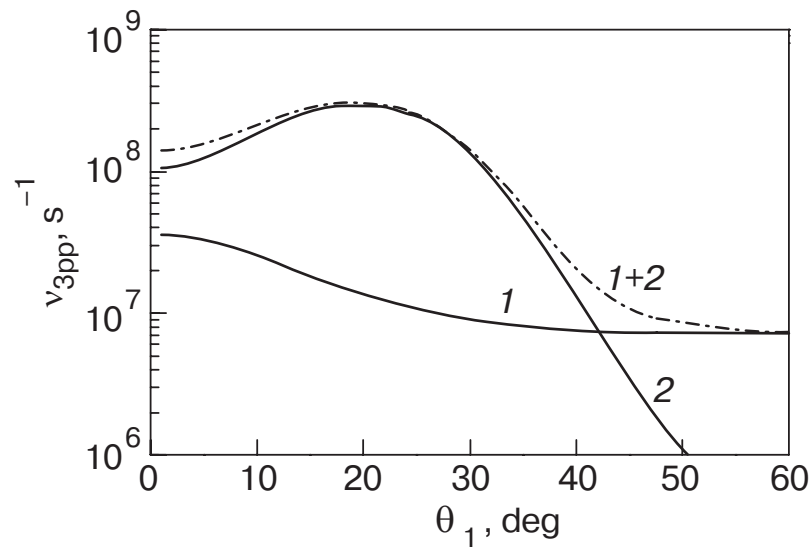

Fig. 5. The dependence of the three-phonon process rate on the angle between the axis of anisotropy and the momentum of the relaxing phonon $\tilde{p}_{1}=\langle\tilde{p}\rangle$ at $P=0$ bar, $T_{p}=$ $=1 \mathrm{~K}$ and $\zeta_{p}=0.023$. Curve 1 shows the rate of decay of a phonon with momentum $\mathbf{p}_{1}$, curve 2 shows the rate of combining of a phonon with momentum $\mathbf{p}_{1}$ with a phonon of the anisotropic phonon system, curve $1+2$ is the sum of curves 1 and 2 and corresponds to the full relaxation rate of a phonon with momentum $\mathbf{p}_{1}$ in the anisotropic phonon system. anisotropic phonon system. At greater values of the angle between phonon momentum and the axis of anisotropy, the phonon ceases «to feel» the presence of the anisotropic phonon system, and as a result there is no angular dependence. In this case the rate of decay corresponds to the rate of spontaneous decay of a phonon. The maximum of curve 2 can be explained in the following way. We replace the anisotropic phonon system by a monochromatic phonon pulse which moves along the $z$ axis. Then the average momentum of phonons in such pulse is equal to $1.581 \mathrm{~K}$ (see Table 2). We calculate the interaction angle of such phonons and see from Table 2 that $\theta_{\text {eff }}=18^{\circ}$. This is in good agreement with the location of the maximum in Fig. 4, $a$. The locations of the maxima of the other curves in Fig. 4, $a$ can be calculated in a similar way and they are given in Table 2. In Fig. $4, b$ is shown the effect of different temperatures at the saturated vapor pressure and $\zeta_{p}=0.023$. Curves in this case have an angular dependence which is similar to that shown in Fig. 4, $a$. The locations of the maxima in this case are 


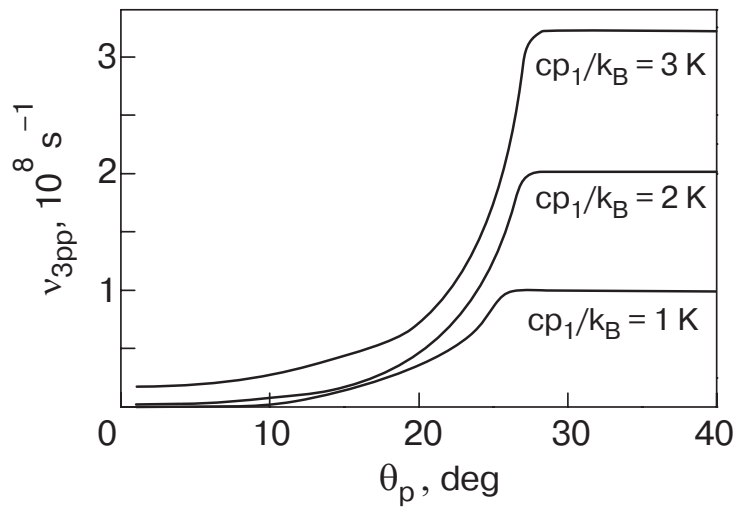

Fig. 6. The dependence of the rate $v_{3 p p}$ on the angle $\theta_{p}$ occupied by the anisotropic phonon system in momentum space for different values of $\tilde{p}_{1}$ at $T=1 \mathrm{~K}$ and $P=0$ bar.

also given in Table 2. Fig. 4,c and 4, $d$ represent the dependence of rate $v_{3 p p}$ on momentum $p_{1}$ at $\theta_{1}=\theta_{\text {eff }}(P, T)$ at different pressures and temperatures. The value of $\zeta_{p}$ was defined by expression (58) (see Table 2). We note that the dependences represented in Fig. 4, $c$ and 4, $d$ look like the dependences of the rates on momentum $p_{1}$ for relaxation in isotropic systems. However in this case the rates are smaller.

The dependence of the rate of three-phonon processes on the value of anisotropy of phonon system can be obtained by calculating the rate of three-phonon processes in anisotropic phonon systems with the distribution function (52) instead of (53). Calculating the rates in this way makes it possible to see the transition from anisotropic phonon system to isotropic. This transition is shown in Fig. 6 where $\theta_{p}$ is the typical angle which the anisotropic phonon system occupies in momentum space. When the angle is zero, there is no anisotropic phonon system. In this case this rate corresponds to the rate of decay of a phonon with momentum $p_{1}$ which was originally obtained in Ref. 21. As the angle increases the rate begins to increase reaching the isotropic value when $\theta_{p} \sim 27^{\circ}$. Such a result is connected with the fact that phonons can interact with each other if the angle between their momenta does not exceed the maximum angle for three-phonon processes. In this case the value of this angle equals to $27^{\circ}$.

\section{Comparison of the theory with experimental data on interaction of phonon pulses}

In experiments $[4,5]$ the interaction of two phonon pulses was studied. In this connection the problem of calculating of the rate of their interaction is of undoubted interest. The calculation of these rates is based on the above solution of the problem of one phonon relaxing in an anisotropic phonon system. In Fig. 7 the dependence of the rate of such an interaction on angle between axes of anisotropy of phonon systems is shown. This angle is shown in the inset in the right upper corner of Fig. 7, $a$ (anisotropic phonon systems are represented by cones). This rate was obtained from relation (60) in the following way: the value of the relaxing phonon momentum was considered to be equal to the average value of momentum of phonons in a pulse, and the averaging over angle was made in order to take into account that actually we are dealing not with one phonon but with an anisotropic phonon system. To average over angles, we took three values of the rate at values of angle $\theta_{\mathbf{u u}^{\prime}}-\theta_{p}, \theta_{\mathbf{u u}^{\prime}}$, $\theta_{\mathbf{u u}^{\prime}}+\theta_{p}$ and calculated their simple average value. We note that such an approach does not take into account spontaneous decays of a phonon with momentum $\mathbf{p}_{1}$, because anisotropic phonon systems by themselves are in equilibrium. However it is necessary to take into account the decay stimulated by the anisotropic phonon system, which, as follows from our calculations (see Fig. 5), gives a small contribution compared to phonons combining processes.

Phonon pulses have enough time to interact if the time to cross the region where the two sheets overlap $\tau_{\text {cross }}$ is greater than the relaxation time of phonons $v_{3 p p}^{-1}$. The expression for $\tau_{\text {cross }}$, according to Ref. 29 , can be written as

$$
\tau_{\text {cross }}=t_{p}\left(2 \sin ^{2}\left(\theta_{\mathbf{u u}^{\prime}} / 2\right)\right)^{-1}
$$

where $t_{p}$ is a duration of the thermal pulse which creates the phonon pulse. In this case in the volume, where the pulses intersect, there is enough time for three-phonon processes to occur and create a new formation, which following the terminology of Ref. 5 we call a hot line.

The formation of a hot line was experimentally observed in [4,5] and it was shown that at high pressures and at large values of angles between pulses, a hot line is not formed. Such a situation can be explained with a help of Fig. 7. At values of angle $\theta_{\mathbf{u u}^{\prime}}$ in experiments $[4,5]$, i.e. $8.8^{\circ}, 26^{\circ}$ and $46^{\circ}$ time $\tau_{\text {cross }}$ at $t_{p}=100 \mathrm{~ns}$ is equal to $8.5 \cdot 10^{-6} \mathrm{~s}, 9.88 \cdot 10^{-7} \mathrm{~s}, 3.28 \cdot 10^{-7} \mathrm{~s}$ respectively. Then as shown in Fig. 7,e, when the pressure is 18 bar, at all values of angle and temperature, the inequality

$$
\tau_{\text {cross }}<v_{3 p p}^{-1}
$$

is satisfied. Thus pulses have no time to interact and a hot line is not formed.

We note also, that at the saturated vapor pressure and at an angle equal to $46^{\circ}$ and $T_{p}=0.7 \mathrm{~K}$ the inequality (67) is satisfied and hot line also has no time to be formed. With a temperature $T_{p}$ of $1 \mathrm{~K}$, the given 

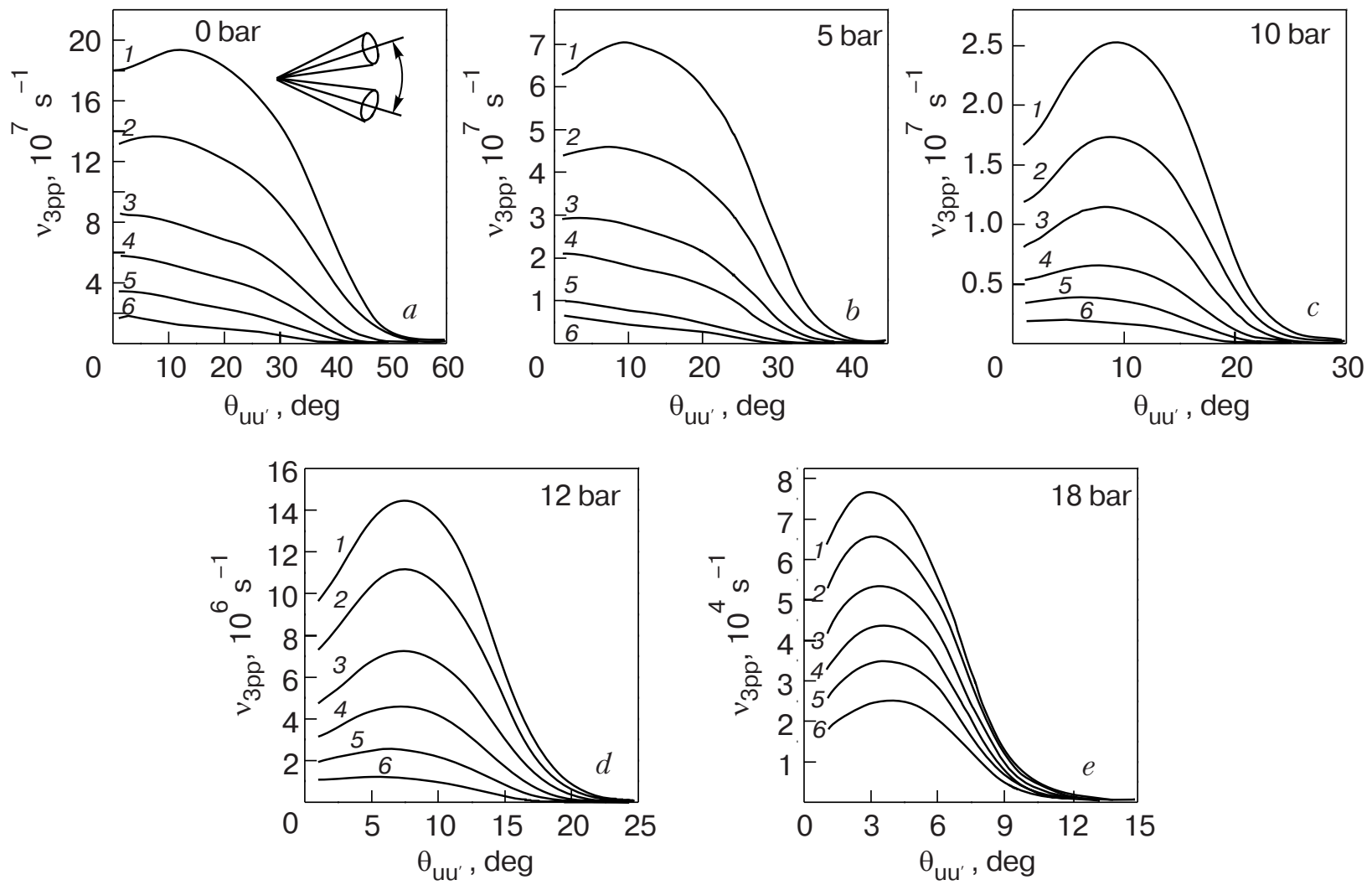

Fig. 7. The rate of three-phonon processes $v_{3 p p}$ as a function of an angle between the axes of the two interacting anisotropic phonon systems. Curves 1-6 of each Fig. are for temperatures $T_{p} 1,0.9,0.8,0.7,0.6$ and $0.5 \mathrm{~K}$. Figures $(a),(b),(c),(d)$, (e) are for pressures $0,5,10,12$ and 18 bar, respectively. The value of $\zeta_{p}$ is defined by Eq. (57) (see Table 2).

angle is about $50^{\circ}$ (see Fig. 7, $a$ ) and again no hotline is formed.

At the saturated vapor pressure, when $\theta_{\text {uu' }}$ equals to $8.8^{\circ}$ and $26^{\circ}$, for all six values of temperature which are represented on Fig. $7, a$ by curves $1-6$ the inequality

$$
\tau_{\text {cross }}>v_{3 p p}^{-1}
$$

is satisfied. In this case pulses have enough time to interact.

The dependence of the interaction of two phonon pulses on angle between their axes at different values of pressure and temperature obtained here, is in full agreement with the results of experiments [4,5].

We note that in experiments $[4,5]$ the angle between pulses $\theta_{\mathbf{u u}^{\prime}}$ and the temperature of the pulses change during motion of pulses from the heater to the detector. An account of these effects was given in Ref. 29. However the comparisons carried out above, answer the question about the possibility of pulses interacting in the conditions of the experiments $[4,5]$.

\section{Conclusion}

In this paper three-phonon processes in isotropic and anisotropic phonon systems of superfluid helium at different pressures were investigated. The approxi- mate dependences (5)-(8) of He II parameters on pressure were obtained. Restrictions on the momenta of the phonons participating in three-phonon processes (see Fig. 1) were studied and analytical expressions (13)-(19) for the boundaries of regions in which three-phonon processes are allowed by the conservation laws of energy and momentum were obtained. The dependence (25) on the angle $\theta_{p^{\prime} p^{\prime \prime}}$ between phonons with momenta $\mathbf{p}^{\prime}$ and $\mathbf{p}^{\prime \prime}$ created as a result of the decay of a phonon with momentum $\mathbf{p}$, using different relations between the momenta of the created phonons, was obtained. This dependence is represented on Fig. 2. The maximum angle (29) of threephonon processes at different pressures was found. Also it was shown, that this angle decreases as pressure incrases.

Starting from the kinetic equation, the expression (39) for the rate of three-phonon relaxation in isotropic phonon systems valid for all phonon energies at which three-phonon processes are allowed by conservation laws at different pressures was obtained. The results of rates evaluated using (39) for different pressures and temperatures, are shown in Fig. 3. Also the analytical expression (45) which describes the behavior of rates in the range $p_{\min }<p_{1}<p_{\max }$ was found. Starting from (45) it was shown that the rates of 
three-phonon processes in a small interval of momentum, reach a maximum value and then rapidly vanish. In this paper the pressure dependence of the rate of three-phonon processes in isotropic phonon systems was investigated, and it was shown that the rates decrease with increasing pressure. The reasons for this decrease were explained. Starting from expression (39) the regions, in which the limiting cases (48) and (51) are valid, were found.

In the paper the relaxation rates (60) in anisotropic phonon systems at different pressures were calculated for the first time. Dependences of these rates on the energy of a relaxing phonon (Fig. 4,c,d) and on the direction of its motion relative to an axis of anisotropy (Fig. $4, a, b$ ), were found. Dependences of the rates on the angle between an axis of anisotropy of the phonon system and on the momentum of the relaxing phonon were investigated, and the presence of a maximum in this dependence was explained. The terms corresponding to combining of a phonon with momentum $\mathbf{p}_{1}$ with a phonon of the anisotropic phonon system and to the decay of a phonon with momentum $\mathbf{p}_{1}$ (see Fig. 5), were compared.

In this paper the transition from an anisotropic phonon system to an isotropic one (Fig. 6) was investigated. It was shown, that starting from some value of $\theta_{p}$, the rate of three-phonon processes becomes saturated.

Based on the solution of a problem of relaxation in anisotropic phonon system, the rates of two interacting anisotropic phonon systems (see Fig. 7) were calculated and compared with experimental data [4,5].

In the conclusion we note that three-phonon relaxation causes the dissipative coefficients of He II and superfluid mixtures of ${ }^{3} \mathrm{He}^{-}{ }^{4} \mathrm{He}$ (see, for example, [24]). The pressure dependence of three-phonon processes rate obtained here allows the determination of the change of this contribution with increasing pressure.

We express our gratitude to GFFI of Ukraine (grant N02.07/000372), and to EPSRC of the UK (grant EP/C523199/1) for support this work.

1. A.F.G. Wyatt, N.A. Lockerbie, and R.A. Sherlock, J. Phys. Condens. Matter 1, 3507 (1989).

2. M.A.H. Tucker and A.F.G. Wyatt, J. Phys.: Condens. Matter 6, 2813 (1994).

3. M.A.H. Tucker and A.F.G. Wyatt, J. Low Temp. Phys. 113, 621 (1998).

4. D.H.S. Smith, R. Vovk, C.D.H. Williams, and A.F.G. Wyatt, Submitted to Phys. Rev. B.

5. R. Vovk, C.D.H. Williams, and A.F.G.Wyatt, Phys. Rev. Lett. 91, 235302 (2003).
6. W. Stirling, Proc. 75th Jubilee Conf. on ${ }^{4}$ He. J.G.M. Armitage (ed.), World Scientific, Singapure (1983), p. 109.

7. R.C. Dynes and V. Narayanamurti, Phys. Rev. B12, 1720 (1975)

8. C.H. Aldrich III and D. Pines J. Low Temp. Phys. 25, 677 (1976).

9. C.H. Aldrich III, C.J. Pethick, and D. Pines, J. Low Temp. Phys. 25, 691 (1976).

10. B.M. Abraham, Y. Eckstein, J.B. Ketterson, M. Kuchnir, and P.R. Roach, Phys. Rev. A1, 250 (1970).

11. R.C. Dynes and V. Narayanamurti, Phys. Rev. Lett. 33, 1195 (1974)

12. R. Sridhar, Phys. Rep. 146, 259 (1987).

13. L.P. Pitayevski and Y.B. Levinson, Phys. Rev. B14, 263 (1976)

14. R.A. Sherlock, N.G. Mills, and A.F.G. Wyatt, J. Phys. C: Solid State Phys. 8, 2575 (1975).

15. J. Jackle and K.W. Kehr, Phys. Rev. Lett. 27, 654 (1971).

16. C.I. Um, S.Y. Lee, S.K. Yoo, T.F. George, L.N. Pandey, and I.N. Adamenko, Fiz. Nizk. Temp. 23, 537 (1997) [Low Temp. Phys. 23, 397 (1997)].

17. T.J. Sluckin and R.M. Bowley, J. Phys. C: Solid State Phys. 7, 1779 (1974).

18. M.A.H. Tucker, A.F.G. Wyatt, I.N. Adamenko, A.V. Zhukov, and K.E. Nemchenko, Fiz. Nizk. Temp. 25, 657 (1999) [Low Temp. Phys . 25, 488 (1999)].

19. I.N. Adamenko, Yu.A. Kitsenko, K.E. Nemchenko, V.A. Slipko, and A.F.G. Wyatt, Abstacts of 11th Int. Conf. on Phonon Scattering in Condensed Matter «Phonons 2004», St. Petersburg (2004), p. 218.

20. Yu.A. Kitsenko and V.A. Slipko, The Journal of Kharkiv National University, Physical Series «Nuclei, Particles, Fields», No. 619, Issue 1(23), 81, (2004) [in Russian].

21. S. Halvin and M. Luban, Phys. Lett. A42, 133 (1972).

22. H.J. Maris, Phys. Rev. A8, 1980 (1973).

23. H.J. Maris, Phys. Rev. A9, 1412 (1974).

24. I.N. Adamenko and E.Ya. Rudavskii, Fiz. Nizk. Temp. 13, 3 (1987) [Low Temp. Phys. 13, 1 (1987)].

25. A.F.G. Wyatt, M.A.H. Tucker, I.N. Adamenko, K.E. Nemchenko, and A.V. Zhukov, Phys. Rev. B62, 9402 (2000)

26. I.N. Adamenko, K.E. Nemchenko, V.A. Slipko, and A.F.G. Wyatt, Phys. Rev. B68, 134507 (2003).

27. A.F.G. Wyatt, M.A.H. Tucker, I.N. Adamenko, K.E. Nemchenko, and A.V. Zhukov, Phys. Rev. B62, 3029 (2000)

28. I.N. Adamenko, K.E. Nemchenko, and A.F.G. Wyatt, Fiz. Nizk. Temp. 29, 16 (2003)] [Low Temp. Phys. 29, 11 (2003)].

29. I.N. Adamenko, Yu.A. Kitsenko, K.E. Nemchenko, V.A. Slipko, and A.F.G. Wyatt, Submitted to Phys. Rev. 